Local behaviour of evaporating stars and black holes around the total evaporation event

This article has been downloaded from IOPscience. Please scroll down to see the full text article.

2010 Class. Quantum Grav. 27125011

(http://iopscience.iop.org/0264-9381/27/12/125011)

View the table of contents for this issue, or go to the journal homepage for more

Download details:

IP Address: 147.83.164.165

The article was downloaded on 02/06/2010 at 12:24

Please note that terms and conditions apply. 


\title{
Local behaviour of evaporating stars and black holes around the total evaporation event
}

\author{
F Fayos ${ }^{1}$ and R Torres \\ Department of Applied Physics, UPC, Barcelona, Spain \\ E-mail: f.fayos@upc.edu and ramon.torres-herrera@upc.edu
}

Received 12 September 2009, in final form 11 February 2010

Published 28 April 2010

Online at stacks.iop.org/CQG/27/125011

\begin{abstract}
Many models in which the object under study loses all its mass have appeared in the literature. This can be found from evaporating stars to incipient black holes, all the way to evaporating black holes. In this paper we try a semiclassical study of these evaporating models centred on the evaporating event itself. We analyse their common properties, behaviours and possibilities. Specifically, we pay special attention to the evaporating models as a means of avoiding singularities during the collapse. In the case of any pre-existing non-spacelike curvature singularity, we show that these models tend to evaporate it. Finally, we introduce a new class of evaporating black holes.
\end{abstract}

PACS numbers: $04.20 . \mathrm{Cv}, 04.40 .-\mathrm{b}, 04.90 .+\mathrm{e}, 98.10 .+\mathrm{z}$

\section{Introduction}

The pioneering work on evaporating models (1968) was an article by Demianski and Lasota [1]. In this work they found an exact solution of Einstein's equations representing a star endowed with a perfect fluid interior with a flux of unpolarized radiation travelling radially outwards. This interior was matched through the evolving stellar surface to a Vaidya solution which modelled the radiating exterior of the star. The proper radius of the model had the particular property of becoming zero in a finite time. Thus, the star evaporated and a flat spacetime appeared in the future of the evaporating event. Later [2], it was shown that this model could be used as a counterexample of Penrose's cosmic censorship conjecture [3] since an instantaneous naked curvature singularity appeared at the evaporating event. Likewise, an evaporating radiating star with a Robertson-Walker interior was proposed in [4] by Lake and Hellaby. This model generates a naked singularity that the authors argue is not instantaneous. However, this is questioned in [5]. The generation of naked singularities by particular models of evaporating stars avoiding the formation of horizons is also treated in [6] where the subject is treated in four and higher dimensions.

1 Also at Laboratori de Física Matemàtica, Societat Catalana de Física, IEC, Barcelona. 
Contrary to the use of evaporating models as a means of getting naked singularities, the ejection of matter until reaching a complete evaporation can also provide a method of avoiding the formation of closed trapped surfaces, singularities (naked or not) and black holes. Thus, for example, in [7], by studying the general procedure for getting spherically symmetric matched models, we obtained a particular analytical example of a regular collapsing toy-star satisfying all the energy conditions which manages to avoid the formation of singularities by radiating away enough mass while the radius of the stellar surface keeps decreasing until becoming zero. In [8] we extended this case to a wide class of models sharing the same properties. Therefore, these models do not need to be in a stationary equilibrium configuration nor to bounce to avoid the formation of a singularity. Along this line, other authors have proposed new evaporating models with different matter fields [9], different exact solutions of Einstein's equations for non-perfect fluids with heat flow [10] or different equations of state [11]. Recently [12], a new mechanism for ejecting matter (based on the mini-super-space loop quantum gravity formalism) has been proposed with the aim of creating this kind of evaporating singularity free models. This is interesting since it indicates that quantum effects could even favour the total evaporation ${ }^{2}$.

Another possibility for evaporating stellar models is that of the incipient black holes. In these models an about-to-be-formed black hole emits radiation until it evaporates without forming neither a black hole nor a singularity. For example, in [13] the black body radiation coming from the collapsing star continuously drains away the irreducible mass of the incipient black hole at an ever increasing rate. The lifetime of the about-to-be-formed black hole is therefore finite and it can never pass through its instantaneous $R=2 \mathrm{M}$ surface. Other recent models for incipient black holes can be found in [14-16], where usually the evaporation of the collapsing object is due to nonthermal pre-Hawking radiation, thus avoiding the information loss problem. Likewise, a treatment of non-singular evaporating black holes in the framework of quantum gravity can also be found in $[17,18]$.

Our goal in this paper is to study the evaporating models as a whole, but paying special attention to the evaporating event itself. We will try to obtain the general conditions that such evaporating objects must satisfy as well as their different behaviours and possibilities using the geometrical approach of requiring the existence of a well-defined metric around the evaporation event. In order to get these models we will match a general interior solution with the exterior radiating Vaidya's solution, in such a way that mass will be lost in the form of outgoing incoherent null radiation. Since the physics of some evaporating models at late stages can be fairly unknown, we will not restrict the energy-momentum tensor in the interior of the model allowing classical or quantum fields. This semiclassical approach will be necessary when we consider regular interiors for evaporating objects reaching high densities and pressures in their final stages. However, even this approach will fail when considering singular interiors in which the second-order curvature invariants diverge. In the vicinity of such singularities only a full quantum gravity theory could provide us with the accurate answers. Therefore, when we deal with this singular case our aim will be to try to discover the possibilities allowed in the semiclassical framework. On the other hand, we will not even impose the fulfilment of any kind of energy conditions in the interior of the models since it is well known that the energy conditions can be violated in the presence of quantum fields. In fact, this is a usual feature for evaporating black holes ([19], proposition 9.2.8).

This work is organized as follows. In section 2 we deal with the radiating Vaidya's solution. We limit the study of this solution to its possibilities as the exterior of an evaporating model and, subsequently, we interpret these possibilities in terms of what is happening with

2 However, some geometrical and physical problems are present. See [8] for details. 
the star. In section 3, we introduce the general interior and study its different properties, characteristics and possibilities around the total evaporation event. In section 4, we study the complete matched models and the dynamical behaviour of the object's surface. The models that lose all its mass without reaching a complete collapse are treated in section 5. In section 6 , we deal with the conditions that should be satisfied by models that collapse and evaporate completely avoiding the formation of any exterior singularity. The models with regular interiors, interiors with a non-spacelike singularity and evaporating black holes are sequentially treated in section 7. Finally, the possible existence of models that develop an exterior singularity in their collapse is studied in section 8 .

\section{Vaidya's solution as the radiating exterior of an evaporating model}

Locally, Vaidya's metric [20] can be described in radiative coordinates [21] as

$$
\mathrm{d} s_{V}^{2}=-\bar{\chi} \mathrm{d} \bar{u}^{2}-2 \mathrm{~d} \bar{u} \mathrm{~d} \bar{R}+\bar{R}^{2}\left(\mathrm{~d} \bar{\theta}^{2}+\sin ^{2} \bar{\theta} \mathrm{d} \bar{\phi}^{2}\right),
$$

where $\bar{\chi}=1-2 \frac{\bar{m}(\bar{u})}{\bar{R}}$ and the mass function $\bar{m}$ [22-24] depends only on $\bar{u}$. For those values of $\bar{u}$ such that $\bar{m}>0$, there is a spacelike curvature singularity at $\bar{R}=0$.

The energy-momentum tensor for metric (1) is of pure radiation type:

$$
\bar{T}_{\mu \nu}=-\frac{2}{\bar{R}^{2}} \frac{\mathrm{d} \bar{m}(\bar{u})}{\mathrm{d} \bar{u}} l_{\mu} l_{\nu}, \quad l_{\mu} \mathrm{d} x^{\mu}=-\mathrm{d} \bar{u}, \quad l_{\mu} l^{\mu}=0 .
$$

The weak energy conditions demand that $\frac{\mathrm{d} \bar{m}(\bar{u})}{\mathrm{d} \bar{u}} \leqslant 0$. We shall assume from now on that $\bar{u}$ grows towards the future. Then, $\bar{m}$ is a non-increasing function of $\bar{u}$ and the incoherent radially directed radiation described by (2) is outgoing. Here, outgoing means going towards bigger values of $\bar{R}$.

We want to describe a process of total evaporation such that the mass function must decrease until vanishing from the total evaporation event on. Vaidya's solution will model the radiating exterior of the evaporating object. Therefore, we are only interested in studying the particular Vaidya's solutions with vanishing mass. We will proceed for the exterior spacetime as follows: we use metric (1) in the interval $-\infty \leqslant \bar{u} \leqslant 0$, assuming that the non-increasing function $\bar{m}(\bar{u})$ satisfies

$$
\bar{m}(\bar{u}=0)=0,
$$

so that we arbitrarily set the evaporation event at $\bar{u}=0$. For $\bar{u} \geqslant 0$ we extend Vaidya's metric with Minkowski's spacetime, i.e. $\bar{m}(\bar{u} \geqslant 0)=0$ (see [7, 8] and references therein for details).

In order to analyse the particular Vaidya's solutions with vanishing mass it is useful to study the behaviour of the radial ingoing null geodesics. To do that, we have to take into account that the differential equation for these null geodesics non-transporting radiation, i.e.

$$
\frac{\mathrm{d} \bar{u}}{\mathrm{~d} \bar{R}}=-\frac{2 \bar{R}}{\bar{R}-2 \bar{m}(\bar{u})},
$$

has a critical point at $\{\bar{R}=0, \bar{u}=0\}$.

In particular, if

$$
-\frac{1}{16} \leqslant \Upsilon \leqslant 0
$$

where

$$
\Upsilon \equiv \lim _{\bar{u} \rightarrow 0^{-}} \frac{\bar{m}(\bar{u})}{\bar{u}}=\lim _{\bar{u} \rightarrow 0^{-}} \frac{\mathrm{d} \bar{m}(\bar{u})}{\mathrm{d} \bar{u}},
$$

then $\bar{u}=\bar{R}=0$ has a node (or col-node) structure $[25,26]$. This implies that every ingoing radial null geodesic must reach the critical point according to a well-defined value for its 


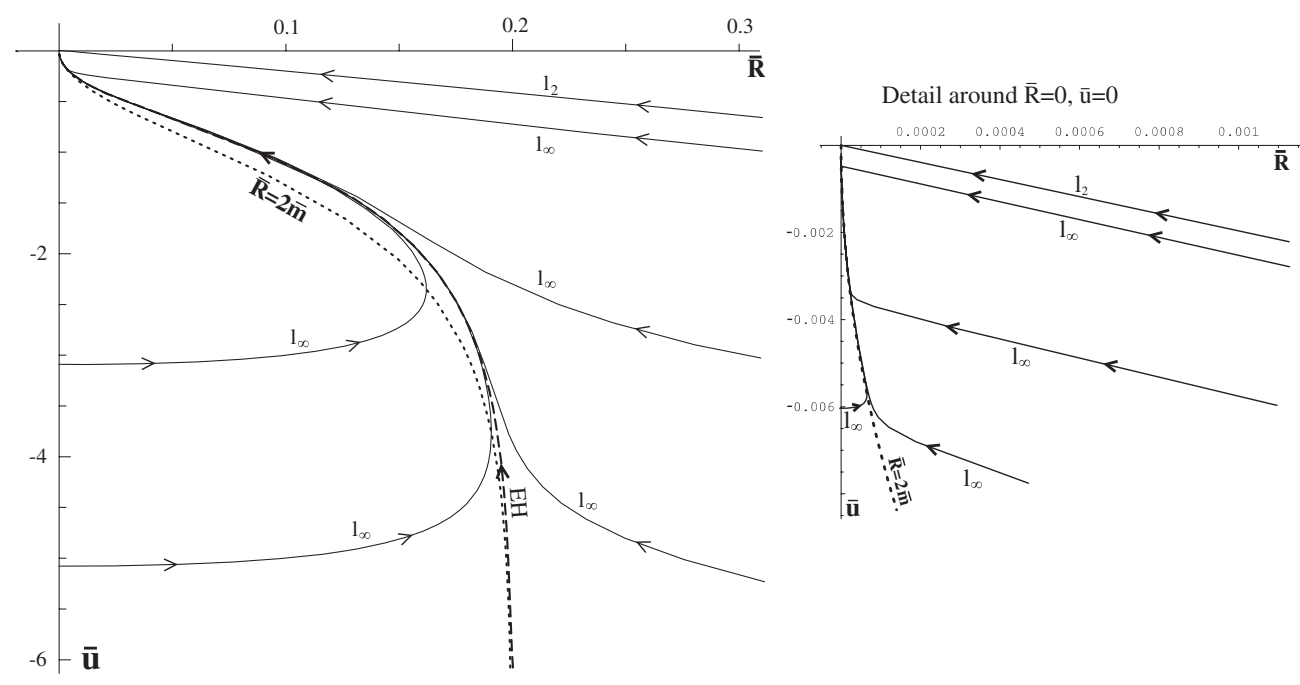

Figure 1. In this graphic we show the behaviour of the ingoing radial null geodesics when $\Upsilon=0$. Note the existence of a null geodesic ending at the critical point with tangent equal to -2 (labelled as $l_{2}$ ) and of null geodesics arriving with minus infinite tangent (labelled as $l_{\infty}$ ).

tangent which can only be one between two possibilities. In order to calculate the values of these tangents, let us define $\xi \equiv \bar{u} / \bar{R}$ and let $\lambda$ be the affine parameter of the radial null geodesic such that $\lim _{\lambda \rightarrow 0} \bar{u}(\lambda)=0$ and $\lim _{\lambda \rightarrow 0} \bar{R}(\lambda)=0$. Then we have, by applying Hôpital's rule, $\left.\xi_{0} \equiv \lim _{\lambda \rightarrow 0} \bar{u} / \bar{R}=\lim _{\lambda \rightarrow 0} \mathrm{~d} \bar{u} / \mathrm{d} \bar{R}=-2 /(1-2 \bar{m}, \bar{u}\rfloor_{\bar{u}=0} \xi_{0}\right)$, which provides us with the two possible values for the critical directions:

$$
\xi_{0 \pm}(\Upsilon)=\frac{1 \pm \sqrt{1+16 \Upsilon}}{4 \Upsilon}
$$

Therefore, condition (5) implies

$$
-\infty<\xi_{0_{+}} \leqslant-4 \leqslant \xi_{0-} \leqslant-2 .
$$

Even more, the node (or col-node) structure implies that there is an infinite family of spherically symmetric null hypersurfaces reaching the critical point $\bar{u}=\bar{R}=0$ with tangent $\xi_{0+}$, while there is only one with tangent $\xi_{0-}[27-29]$ as illustrated in figure 1.

In what follows, it will be useful to know the values of $\bar{\chi}$ at $\bar{u}=\bar{R}=0$ as measured in these two directions $\xi_{0 \pm}$. Using (7)

$$
\bar{\chi}_{0 \pm}(\Upsilon)=\lim _{\bar{u} \rightarrow 0^{-}}\left(1-2 \frac{\bar{m}(\bar{u})}{\bar{R}}\right)=\frac{1}{2}(1 \mp \sqrt{1+16 \Upsilon}),
$$

which implies

$$
0 \leqslant \bar{\chi}_{0+}(\Upsilon) \leqslant \frac{1}{2} \leqslant \bar{\chi}_{0-}(\Upsilon) \leqslant 1
$$

In particular, if $\Upsilon=0$ then $\xi_{0+}=-\infty, \bar{\chi}_{0+}=0, \xi_{0-}=-2$ and $\bar{\chi}_{0-}=1$.

The translation of these results into Penrose's diagrams is shown in figures 2 and 3 , where a null singularity $\bar{u}=\bar{R}=0$ appears. In practice, only if we know the behaviour of $\bar{m}(\bar{u})$ for all $\bar{u}$ in the interval $(-\infty, 0)$ we will be able to distinguish between the two possibilities appearing in the figures.

For the sake of completeness, let us remark that if $\Upsilon<-\frac{1}{16}$ the critical point in the differential equation for the radial ingoing null geodesics is an unstable focus. The 


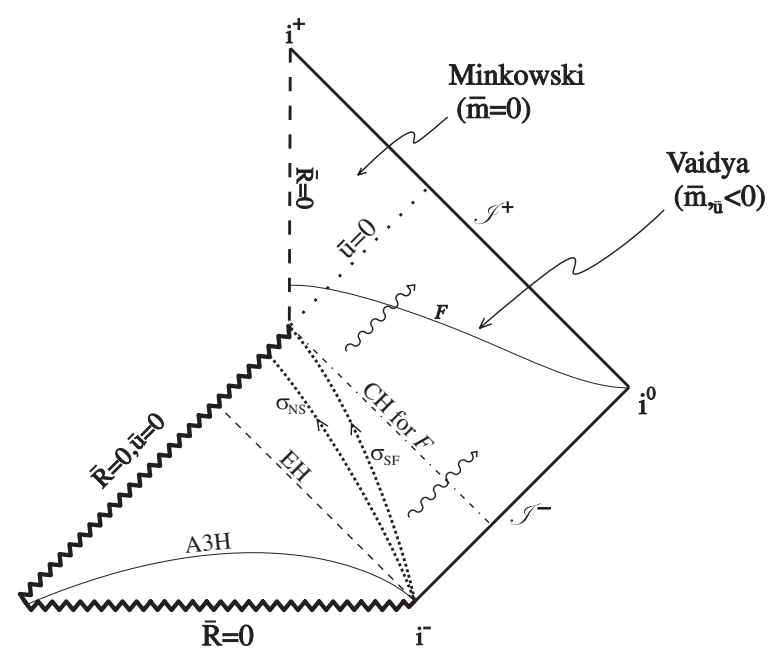

Figure 2. The Penrose diagram for Vaidya's spacetime when $\bar{m}(\bar{u})$ vanishes from some instant $\bar{u}=0$ on and $-1 / 16 \leqslant \Upsilon \leqslant 0$. Note that in this diagram some ingoing radial null geodesics come from the past null infinity $\mathcal{J}^{-}$and reach the light-like singularity $(\bar{R}=0, \bar{u}=0)$. A generic spacelike hypersurface $F$ has the past Cauchy horizon in the null hypersurface marked as ' $\mathrm{CH}$ for $F^{\prime}$.

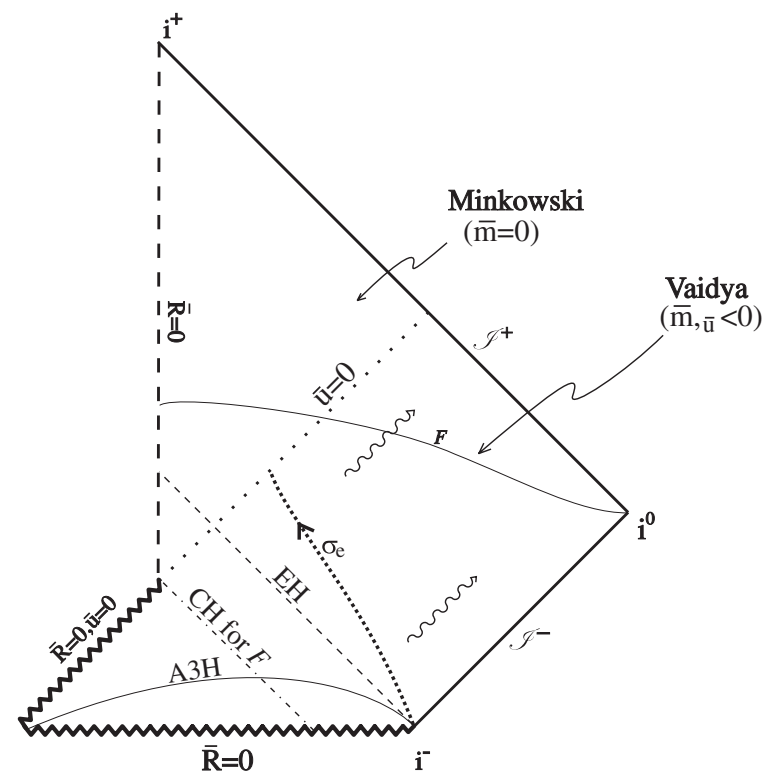

Figure 3. Alternative Penrose's diagram for Vaidya's spacetime when $\bar{m}(\bar{u})$ vanishes from some instant $\bar{u}=0$ on and $-1 / 16 \leqslant \Upsilon \leqslant 0$. Note that in this diagram no ingoing radial null geodesic comes from the past null infinity $\mathcal{J}^{-}$and reaches the light-like singularity. A generic spacelike hypersurface $F$ has the past Cauchy horizon in the null hypersurface marked as ' $\mathrm{CH}$ for $F$ '.

Penrose diagram corresponding to this case can be seen in figure 4 . No other cases exist since $\Upsilon \leqslant 0$. 


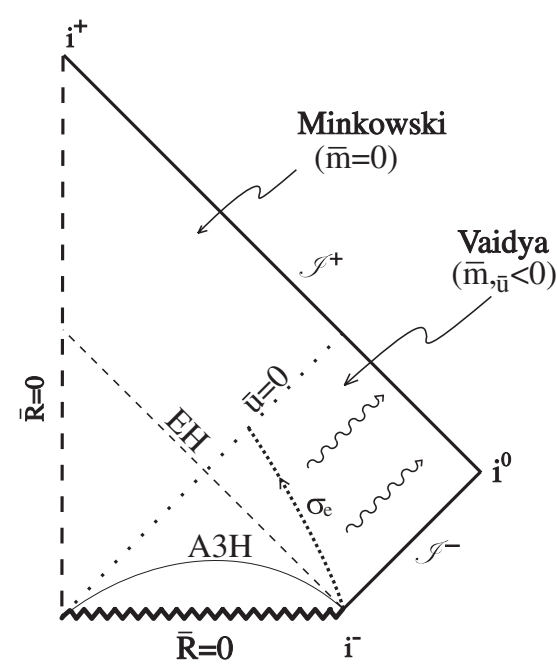

Figure 4. The Penrose diagram for Vaidya's solution when $\bar{m}(\bar{u})$ vanishes from some instant $\bar{u}=0$ on and $\Upsilon<-1 / 16$.

\subsection{Candidates for matching the hypersurface in Vaidya's spacetime}

The behaviour of the stellar model will depend both on the chosen solution for the interior and on the hypersurface chosen as the matching hypersurface. In this way, a totally collapsing evaporating model must have a timelike matching hypersurface $\Sigma$ ending at $\bar{u}=\bar{R}=0$. This exclusively corresponds with hypersurfaces of types $\sigma_{\mathrm{NS}}$ and $\sigma_{\mathrm{SF}}$ appearing in figure $2 .{ }^{3} \mathrm{In}$ the case $\sigma_{\mathrm{NS}}$ a null singularity emerges beyond the total evaporation event, while this does not happen if the matching hypersurface were $\sigma_{\mathrm{SF}}$.

A spherically symmetric timelike hypersurface $(\bar{u}(\tau), \bar{R}(\tau))$ (where $\tau$ is a future-directed time coordinate defined only on the hypersurface) is timelike and future directed if, and only if,

$$
\bar{\chi} \dot{\bar{u}}+2 \dot{\bar{R}}>0
$$

and $\dot{\bar{u}}>0$, where dots stand for derivatives with respect to $\tau$.

The slope of such hypersurface when reaching $\bar{u}=0, \bar{R}=0$, at let us say $\tau=0$, in the $\{\bar{u}, \bar{R}\}$ plane can be computed as

$$
\zeta \equiv \lim _{\tau \rightarrow 0^{-}} \frac{\bar{u}(\tau)}{\bar{R}(\tau)}=\lim _{\tau \rightarrow 0^{-}} \frac{\dot{\bar{u}}}{\dot{\bar{R}}} .
$$

Likewise, the value of $\bar{\chi}$ on the hypersurface as it approaches $\bar{u}=0, \bar{R}=0$ will be

$$
\bar{\chi}_{0 \zeta} \equiv \lim _{\tau \rightarrow 0^{-}}\left(1-2 \frac{\bar{m}(\bar{u})}{\bar{R}}\right)=1-2 \Upsilon \zeta .
$$

If we use (11)-(13), we find that the slope of the timelike hypersurface reaching $\bar{u}=\bar{R}=0$ must satisfy

$$
-\infty<\xi_{0_{+}}(\Upsilon) \leqslant \zeta \leqslant \xi_{0-}(\Upsilon) \leqslant-2
$$

where $\xi_{0 \pm}$ are defined in (7) and we have taken into account that, since the timelike hypersurface reaches $\bar{R}=\bar{u}=0$ which does not belong to the manifold, the limiting value could also be

3 This type of hypersurfaces does not exist in the particular Vaidya's solutions shown in figures 3 and 4 . 
$\xi_{0 \pm} \cdot{ }^{4}$ Let us remark that inequality (14) is expected from the structure shown for the ingoing radial null geodesics.

If we now use (7) and (13), we find the following bounds for $\bar{\chi}_{0 \zeta}$ :

$$
0 \leqslant \bar{\chi}_{0+}(\Upsilon) \leqslant \bar{\chi}_{0 \zeta}(\Upsilon, \zeta) \leqslant \bar{\chi}_{0-}(\Upsilon) \leqslant 1 .
$$

When dealing with a spherically symmetric timelike hypersurface ending at $\bar{u}=\bar{R}=0$ (see figure 2), a way of classifying it as type $\sigma_{\mathrm{NS}}$ or type $\sigma_{\mathrm{SF}}$ is to note that only timelike hypersurfaces of type $\sigma_{\mathrm{SF}}$ cross all the ingoing radial geodesics near the Cauchy horizon for $F$ (except for this same $\mathrm{CH}$ ). Due to the node (or col-node) structure of the family of ingoing radial null geodesics, in the $\{\bar{R}, \bar{u}\}$ plane (see figure 1) this means that a curve of type $\sigma_{\mathrm{NS}}$ must fulfil

$$
\begin{aligned}
& \zeta_{\mathrm{NS}}=\xi_{0+}(\Upsilon) \\
& 0 \leqslant \bar{\chi}_{0 \zeta_{\mathrm{NS}}}\left(\Upsilon, \zeta_{\mathrm{NS}}\right)=\bar{\chi}_{0+}(\Upsilon) \leqslant \frac{1}{2}
\end{aligned}
$$

We would like to remark that this is the behaviour of, for example, all radial timelike geodesic hypersurfaces ending at the singularity $\bar{u}=\bar{R}=0$ [29].

On the other hand, it is sufficient for a curve to be of the type $\sigma_{\mathrm{SF}}$ to fulfil

$$
\begin{aligned}
& \xi_{0_{+}}(\Upsilon)<\zeta_{\mathrm{SF}} \leqslant \xi_{0_{-}}(\Upsilon) \\
& 0 \leqslant \bar{\chi}_{0+}(\Upsilon)<\bar{\chi}_{0 \zeta_{\mathrm{SF}}}\left(\Upsilon, \zeta_{\mathrm{SF}}\right) \leqslant \bar{\chi}_{0-}(\Upsilon) \leqslant 1
\end{aligned}
$$

Another possibility for evaporation is possible if the matching hypersurface is of type $\sigma_{e}$, as can be seen in figures 3 and 4 . In this case the stellar surface would reach $\bar{u}=0$, corresponding to $\bar{m}(\bar{u}=0, \bar{R} \neq 0)=0$, with a non-zero value of $\bar{R}\left(\bar{R} \equiv \bar{R}_{0}\right)$. This means that from $\bar{u}=0$ on, the mass-energy contained in the sphere $\bar{R}=\bar{R}_{0}$ vanishes [23]. Beyond this point $\Sigma$ could be continued with an arbitrary timelike trajectory crossing the Minkowski region. Therefore, no singularities can be formed there.

\section{The interior solution}

Let us consider a four-dimensional spherically symmetric spacetime $\mathcal{V}$, so that its line element can be expressed in radiative coordinates $[21]\left\{x^{\mu}\right\}=\{u, R, \theta, \varphi\}(\mu=0,1,2,3)$ as

$$
\mathrm{d} s^{2}=-\mathrm{e}^{4 \beta} \chi \mathrm{d} u^{2}+2 \varepsilon \mathrm{e}^{2 \beta} \mathrm{d} u \mathrm{~d} R+R^{2} \mathrm{~d} \Omega^{2},
$$

where $\chi \equiv 1-2 m / R, \varepsilon^{2}=1, \beta$ and $m$ depend on $\{u, R\}$, and $\mathrm{d} \Omega^{2} \equiv \mathrm{d} \theta^{2}+\sin ^{2} \theta \mathrm{d} \varphi^{2}$. It is easily checked that $m(u, R)$ is the mass function [22-24], which represents the total energy inside the two-spheres with constant values of $u$ and $R$.

We choose $u$ growing to the future; then

$$
\mathbf{l}=\frac{\mathrm{d}}{\mathrm{d} \ell}=-\varepsilon \frac{\partial}{\partial R}
$$

is a future-directed radial null vector tangent to the null geodesics $u=$ constant and $\ell$ is a future-directed parameter. If $\varepsilon=-1$ (or +1 ), then the expansion of these null geodesics is positive (negative, respectively) and, therefore, they are outgoing (ingoing, respectively) radial null geodesics. On the other hand,

$$
\mathbf{k}=\frac{\mathrm{d}}{\mathrm{d} \kappa}=\mathrm{e}^{-2 \beta} \frac{\partial}{\partial u}+\varepsilon \frac{\chi}{2} \frac{\partial}{\partial R}
$$

4 Note that, nevertheless, the hypersurface would be timelike in the manifold. 
is a future-directed radial null vector such that $\mathbf{l} \cdot \mathbf{k}=-1$ and $\kappa$ is a future-directed parameter. If $\chi>0$ and $\varepsilon=-1$ (or +1 ), then this vector is tangent to a family of null geodesics with negative (positive, respectively) expansion and, therefore, these radial null geodesics are ingoing (outgoing, respectively). It is interesting to note that if, in a given 2 -sphere, $\chi<0$ and $\varepsilon=-1(\varepsilon=+1)$ then the two radial null vectors have both positive (negative) expansion which means that the 2 -sphere is a closed surface trapped to its past (future, respectively). We will call the spherically symmetric hypersurface defined by $\chi=0$ the apparent 3-horizon, A3H for short.

It is well known that spacetimes can be classified according to the properties of the energy-momentum tensor [19]. A spherically symmetric spacetime is said to be type I if its energy-momentum tensor diagonalizes in an orthonormal basis $E_{0}$ (timelike), $E_{1}, E_{2} \propto \partial / \partial \theta$ and $E_{3} \propto \partial / \partial \varphi$, being $\lambda_{\alpha}$ the corresponding eigenvalues, as long as

$$
\alpha \equiv \beta,,_{R}\left(\chi^{2} \beta,_{R}+\varepsilon \frac{2 \mathrm{e}^{-2 \beta}}{R} m,,_{u}\right)>0
$$

is satisfied. The corresponding eigenvalues are

$$
\begin{aligned}
& \lambda_{0} \equiv-\rho_{d}=-2 \frac{m,_{R}}{R^{2}}+\frac{2}{R}\left(\chi \beta,_{R}-\alpha^{1 / 2}\right), \\
& \lambda_{1} \equiv p_{d}=-2 \frac{m,_{R}}{R^{2}}+\frac{2}{R}\left(\chi \beta,_{R}+\alpha^{1 / 2}\right), \\
& \lambda_{2} \equiv p_{T}=\left(\frac{3-\chi}{R}+4 \chi \beta,_{R}-\frac{6}{R} m,_{R}\right) \beta,_{R}+2 \chi \beta,_{R R}-\frac{1}{R} m,_{R R}+\varepsilon 2 \mathrm{e}^{-2 \beta} \beta,_{u R}, \\
& \lambda_{3}=\lambda_{2} .
\end{aligned}
$$

If $\alpha=0$ then $\lambda_{0}=\lambda_{1}=-2 m,,_{R} / R^{2}$ and we have a double eigenvalue that corresponds to the null eigenvector $\mathbf{l}$. Then the energy-momentum tensor is said to be type II.

The dominant energy conditions (DECs) [19] require the energy density and the energy flux measured by any timelike observer to be non-negative and non-spacelike, respectively. These conditions applied to our interior line element written in radiative coordinates can be written, in both (type I and II) cases, as

$$
\begin{aligned}
& \beta,_{R} \geqslant 0, \\
& m,_{R}-R \chi \beta,,_{R} \geqslant 0, \\
& \chi^{2} \beta,_{R}+\varepsilon \frac{2 \mathrm{e}^{-2 \beta}}{R} m,,_{u} \geqslant 0, \\
& \rho_{d}-p_{T} \geqslant 0, \\
& \rho_{d}+p_{T} \geqslant 0 .
\end{aligned}
$$

Let us now consider a first application of the DEC that we will use later.

Proposition 3.1. In regions fulfilling the DECs and with $\chi>0$

- the mass function must be a non-decreasing function along the outgoing radial null geodesics;

- the mass function must be a non-increasing function along the ingoing radial null geodesics.

To show this we can use (28) and (29) and the condition $\chi>0$ to get

$$
m,,_{R} \geqslant 0,
$$


and we can use (29) and (30) and the condition $\chi>0$ to get

$$
\frac{\chi}{2} m,_{R}+\varepsilon \mathrm{e}^{-2 \beta} m,_{u} \geqslant 0 .
$$

On the other hand, the variation of the mass function along a $u=$ constant null geodesic can be computed using (21) to get

$$
\frac{\mathrm{d} m}{\mathrm{~d} \ell}=-\varepsilon m, R .
$$

And the variation of the mass function along the other family of radial null geodesics can be computed using (22) to get

$$
\frac{\mathrm{d} m}{\mathrm{~d} \kappa}=\varepsilon\left(\frac{\chi}{2} m,,_{R}+\varepsilon \mathrm{e}^{-2 \beta} m,,_{u}\right) .
$$

For outgoing radial null geodesics, if $\varepsilon=-1$ then $\mathbf{l}$ is the tangent to these geodesics and we must combine (33) and (35) to show that the mass is non-decreasing along the outgoing geodesics. However, if $\varepsilon=+1$ then $\mathbf{k}$ is now the vector tangent to the outgoing geodesics and we must combine (34) and (36) to show that the mass is non-decreasing along the outgoing geodesics. (It is enough to follow a similar reasoning to show that along ingoing radial null geodesics the mass function will be non-increasing).

\subsection{Inner local behaviour}

Let us consider $\mathcal{U} \equiv\{(u, R) \mid \delta u \leqslant u \leqslant 0,0 \leqslant R \leqslant \delta R\}$, where the boundaries $\delta u$ and $\delta R$ must be fixed according to some natural conditions for evaporating models that will be stated from section 6 on. In order to build the Einstein field equations we need $\beta$ and $m$ to be $C^{2}$ in the points of $\mathcal{U}$ belonging to the manifold. In this paper we will assume that $\beta$ and $m$ are $C^{2}$ in $\mathcal{U}$.

Without loss of generality, we will always consider $\beta(u, R=0) \equiv \beta_{0}(u)=0$ (since, if it was not, we can always perform a coordinate change $u \rightarrow u^{\prime}$ such that the new coordinates $u^{\prime}$ were defined by $\left.\mathrm{d} u^{\prime}=\mathrm{e}^{2 \beta_{0}(u)} \mathrm{d} u\right)$.

According to the values of $m(u, R)$ and $\beta(u, R)$ and their partial derivatives computed at $u=R=0$ we can classify $\mathcal{U}$ (with a fixed characterization of $R=0$ ) as follows.

(1) Regular interiors. If there are no curvature singularities in $\mathcal{U}$, i.e. if the curvature scalar invariants polynomial in the Riemann tensor (henceforth second-order curvature invariants) remain finite at $\mathcal{U}$ : A full independent set of invariants was found in [30]. By using this set it is easy to show that, out of $R=0$, we have a singularity free region of the spacetime if, and only if, $\beta$ and $m$ and their first and second partial derivatives are non-diverging functions there, but this is guaranteed by our assumption that both $m$ and $\beta$ are $C^{2}$. On the other hand, one gets some conditions on $R=0$ which lead to the following statement [31, 32]: all curvature invariants will be finite at $\mathcal{U}$ preventing the existence of curvature singularities if, and only if,

$$
\lim _{R \rightarrow 0} \frac{\beta(u, R)}{R^{2}}=\beta_{2}(u) \quad \text { and } \quad \lim _{R \rightarrow 0} \frac{m(u, R)}{R^{3}}=m_{3}(u),
$$

where $\beta_{2}(u)$ and $m_{3}(u)$ are finite functions in $\mathcal{U}$. This implies that

$$
\begin{aligned}
& m(u, R=0)=m,_{R}(u, R=0)=m,_{R R}(u, R=0)=0 \\
& \beta(u, R=0)=\beta,_{R}(u, R=0)=0 .
\end{aligned}
$$


(2) Interiors with a non-spacelike singularity at $R=0$. It is easy to show that we have a non-spacelike singularity at $R=0$ if, and only if, conditions (38) are not all fulfilled but

$$
m(u, R=0)=0 \quad \text { and } \quad m,,_{R}(u, R=0) \leqslant 1 / 2 .
$$

(3) Interiors with a spacelike singularity at $R=0$. In this case

$$
m(u, R=0)>0 \quad \text { or } \quad m(u, R=0)=0 \quad \text { and } \quad m,_{R}(u, R=0)>1 / 2 .
$$

It should be noted (37) that in cases (2) and (3) the second-order curvature invariants diverge.

A spherically symmetric timelike hypersurface $\{u(\tau), R(\tau)\}$ (where $\tau$ is a future-directed time coordinate defined only on the hypersurface) is timelike and future directed in the manifold if, and only if,

$$
2 \varepsilon \dot{R}-\mathrm{e}^{2 \beta} \chi \dot{u}<0
$$

and $\dot{u}>0$, where dots stand for derivatives with respect to $\tau$. However, we need to remark that in case the hypersurface reaches a singularity at $R=0$, then the inequalities in the timelike conditions above could be replaced by equalities in the limit as $R$ tends to 0 while the hypersurface would be timelike in the manifold.

Whenever a mass function satisfies $m(u=0, R=0)=0$ and there is a spherically symmetric hypersurface reaching $u=R=0$, in general the value of $\chi$ computed along this hypersurface in the limit when it reaches $u=R=0$ will depend on the specific hypersurface. However, there will be certain important situations in which the value of $\chi$ in this limit is fixed. Consider the following.

Proposition 3.2. If a mass function $m$ is $C^{2}$ in $\mathcal{U}$ and satisfies $m(\delta u \leqslant u \leqslant 0, R=0)=0$ $(\delta u \neq 0)$ and $m,,_{R}(0,0)=0$, then the limiting value of $\chi$ along an arbitrary spherically symmetric hypersurface $\{u(\tau), R(\tau)\}$ reaching $u=R=0$ at $\tau=0$ satisfies

$$
\lim _{\tau \rightarrow 0^{-}} \chi=1 \text {. }
$$

In order to show this, consider in $\mathcal{U}$ a first-order power-series development in $R$ of the mass function taking into account that $m(\delta u \leqslant u \leqslant 0, R=0)=0$ :

$$
m(u, R)=m,_{R}(u, R=0) R+\frac{1}{2} m,_{R R}(u, R=\rho) R^{2},
$$

where the last term is the remainder and, according to Taylor's theorem, $0 \leqslant \rho \leqslant R$. Likewise, $m,,_{R}(u, R=0)$ can be developed in $u$ taking into account that $m,,_{R}(0,0)=0$ to obtain the following zeroth-order power-series development:

$$
m,_{R}(u, R=0)=m,_{u R}(u=v, R=0) u,
$$

where only the remainder appears and, according to Taylor's theorem, $u \leqslant v \leqslant 0$. Finally, with (43) and (44) and using $u(\tau=0)=0$ and $R(\tau=0)=0$ we get

$$
\begin{aligned}
\lim _{\tau \rightarrow 0^{-}} \chi & =1-2 \lim _{\tau \rightarrow 0^{-}} \frac{m}{R} \\
& =1-2 \lim _{\tau \rightarrow 0^{-}}\left(m,{ }_{u R}(u=v, R=0) u+\frac{1}{2} m, R R(u, R=\rho) R\right)=1 .
\end{aligned}
$$

\section{The complete matched models}

Now, in order to get the complete evaporating models, we need to match a part of the interior spacetime containing $R=0$ with the exterior radiating Vaidya's solution through a spherically 
symmetric timelike matching hypersurface identified with the evolving star's surface. In fact, we are interested in the local behaviour around the evaporating event. Therefore, we restrict the analysis of our matching to $\mathcal{U}$ in the interior manifold and to $\overline{\mathcal{U}}$ in the Vaidya spacetime, where similarly to $\mathcal{U}$, we define $\overline{\mathcal{U}} \equiv\{(\bar{u}, \bar{R}) \mid \delta \bar{u} \leqslant \bar{u} \leqslant 0,0 \leqslant \bar{R} \leqslant \delta \bar{R}\}$ and the boundaries must be fixed according to the conditions that we will discuss from section 6 on.

The matching of two spherically symmetric spacetimes through a timelike matching hypersurface $\Sigma$ can be found in [7] and references therein. First we need to identify an embedding of $\Sigma$ in both the Vaidya and the general interior spacetimes. To that end let us consider a general timelike hypersurface $\bar{\sigma}$ preserving the spherical symmetry of the exterior spacetime and with intrinsic coordinates $\left\{\xi^{a}\right\}=\{\lambda, \vartheta, \varphi\}$, where $\lambda$ is a future-directed time coordinate defined only on the hypersurface. The general parametric equations of $\bar{\sigma}$ are: $\bar{u}=\bar{u}(\lambda), \bar{R}=\bar{R}(\lambda), \bar{\theta}=\vartheta, \bar{\phi}=\varphi$. Similarly, let us take a general spherically symmetric timelike hypersurface $\sigma$ in the interior spacetime. This hypersurface is assumed to be diffeomorphic to $\bar{\sigma}$, and thus the intrinsic coordinates are chosen to be the same $\left\{\xi^{a}\right\}=\{\lambda, \vartheta, \varphi\}$. The general parametric equations for $\sigma$ are $u=u(\lambda), R=R(\lambda), \theta=\vartheta, \phi=\varphi$.

Finally, the Darmois gravitational junction conditions (see [7] and references therein for details) applied to the case $\varepsilon=-1$ provide us with the following matching conditions:

$$
\begin{aligned}
& \bar{R} \stackrel{\Sigma}{=} R, \\
& \bar{m} \stackrel{\Sigma}{=} m, \\
& \dot{\bar{u}} \stackrel{\Sigma}{=} \mathrm{e}^{2 \beta} \dot{u}, \\
& 0 \stackrel{\Sigma}{=}\left(\chi \beta,,_{R}-\frac{m,_{R}}{2 R}\right) \mathrm{e}^{2 \beta} \dot{u}+\beta,,_{R} \dot{R} .
\end{aligned}
$$

On the other hand, if $\varepsilon=+1$ the matching conditions are

$$
\begin{aligned}
& \bar{R} \stackrel{\Sigma}{=} R, \\
& \bar{m} \stackrel{\Sigma}{=} m, \\
& \bar{\chi} \dot{\bar{u}} \stackrel{\Sigma}{=} \chi \mathrm{e}^{2 \beta} \dot{u}-2 \dot{R}, \\
& 0 \stackrel{\Sigma}{=}\left[\chi\left(\chi \beta,,_{R}-\frac{m,,_{R}}{2 R}\right)+\frac{m,_{u} \mathrm{e}^{-2 \beta}}{R}\right] \mathrm{e}^{2 \beta} \dot{u}+\left(\frac{m,_{R}}{R}-\chi \beta, R\right) \dot{R} .
\end{aligned}
$$

We choose $\bar{u}(\lambda=0)=u(\lambda=0)=0$, so that $\lambda=0$ is identified with the total evaporation event.

Regarding the existence of two sets of matching conditions, it is interesting to note that the choice $\varepsilon=-1$ implies that an outgoing radial null geodesic defined by $u=$ constant when crossing $\Sigma$ is simply continued with an outgoing radial null geodesic in the Vaidya metric defined by $\bar{u}=$ constant. Contrarily, if we choose $\varepsilon=+1$ a radial ingoing null geodesic defined by $u=$ constant is continued through $\Sigma$ with a radial ingoing null geodesic in the Vaidya metric which is not defined by $\bar{u}=$ constant. This has as a consequence that the analysis of the $\varepsilon=+1$ case is generally more complicated.

\section{Evaporating models that do not completely collapse}

We first study the evaporating models that lose all their mass without completely collapsing. The exterior for these models was studied in subsection 2.1. There we saw that the matching 


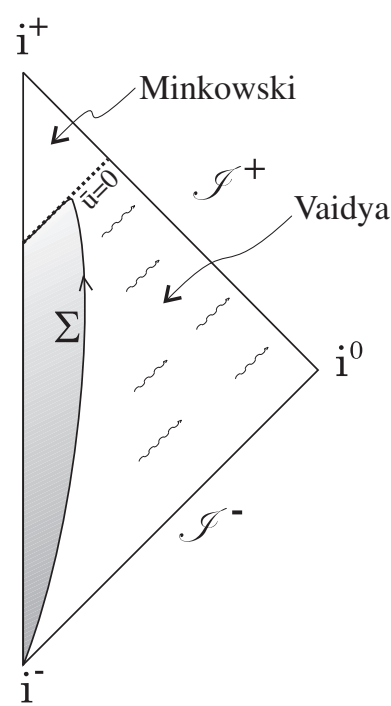

Figure 5. An evaporating model that loses all its mass without completely collapsing.

hypersurface must be of type $\sigma_{e}$ (see, for example, figure 4). In these models, the following proposition applies.

Proposition 5.1. Let a model be such that it fulfils the DECs, the 2-spheres of the model are not closed trapped surfaces $(\chi>0)$ and the mass function is not negative. Then, if the mass function vanishes on the matching hypersurface at, let us say, $u=\bar{u}=0$ and $R \stackrel{\Sigma}{=} \bar{R} \stackrel{\Sigma}{=} \bar{R}_{0}>0$, the matched model will have null mass on and in the future of the outgoing spherically symmetric light-like hypersurface that, starting at $R=0$ and ending at the future null infinity, crosses $\Sigma$ at $\left(u=0, R=\bar{R}_{0}\right)$.

This behaviour has been illustrated in figure 5. Let us remark that the boundary of the region in the interior solution defined by $m=0$ does not need to coincide with the light-like hypersurface introduced in the proposition.

In order to show proposition 5.1, we must consider the outgoing radial null geodesic reaching the matching hypersurface at $\left(u=0, R=\bar{R}_{0}\right)$, where the mass function becomes null. According to proposition 3.1, the mass function must be null along the outgoing radial null geodesic from $R=0$ until reaching $\left(u=0, R=\bar{R}_{0}\right)$. Moreover, as $\bar{m}(\bar{u}=0)=0$, the mass function will be null along $\bar{u}=0$ in the exterior region and, thus, it will be null along all the geodesic. We can always match the model across this null geodesic with a portion of Minkowski's solution. (See [33] for details). In this way we get a model of a completely evaporating star that vanishes without generating any singularity.

\section{Evaporating collapsing models: the case $\Sigma$ of type $\Sigma_{\mathrm{SF}}$}

In this section, we will analyse the matching in the cases in which the matching hypersurface reaches $R=u=0=\bar{R}=\bar{u}$ with $\bar{m}(\bar{u} \geqslant 0)=0$, in such a way that no exterior singularities appear. That is, the cases in which $\Sigma$ will be identified with a hypersurface of type $\sigma_{\mathrm{SF}}$ in Vaidya's spacetime and, as stated in subsection 2.1, (18) and (19) hold. Henceforth, a matching 
hypersurface of this type will be denoted by $\Sigma_{\mathrm{SF}}$. We will list the sufficient conditions for a general interior to produce this kind of evaporating collapse: 'the evaporating collapsing conditions'. The analysis of every particular interior and the necessary and sufficient conditions for every case will be treated in the next section.

\subsection{The evaporating collapsing conditions on the exterior}

(1) There must be a $C^{1}$ solution $\bar{R}(\lambda)$ to the matching conditions in $\overline{\mathcal{U}}$ satisfying

$$
\begin{aligned}
& \bar{R}(\lambda=0)=0, \\
& \bar{R}(\lambda<0)>0 .
\end{aligned}
$$

(2) We demand the mass of the object to be non-negative according to an observer in the exterior:

$$
\bar{m}(\bar{u}<0)>0 \quad \text { and } \quad \bar{m}(\bar{u}=0)=0 .
$$

(3) The matching hypersurface must be timelike in the manifold

$$
\frac{\dot{\bar{R}}}{\dot{\bar{u}}}+\frac{\bar{\chi}}{2} \stackrel{\Sigma_{\mathrm{SF}}}{>} 0
$$

in $\overline{\mathcal{U}}-\{\bar{u}=\bar{R}=0\}$.

(4) As stated in subsection 2.1, the matching with $\Sigma$ of type $\Sigma_{\mathrm{SF}}$ imposes a bound on $\Upsilon$ :

$$
-\frac{1}{16} \leqslant \Upsilon \leqslant 0
$$

It is appropriate to remark now that we will also require a condition on the interior. In the case of existence of a previous spacelike singularity in the interior spacetime the presence of a region in which the 2 -spheres are closed surfaces trapped to their past $(\varepsilon=-1)$ has been excluded. In this way, we avoid, for example, the presence of a white hole in a stellar interior. Thus, we only treat the case $\varepsilon=+1$ in this situation.

\subsection{Consequences of the conditions}

- From 1. $\dot{R} / \dot{u}$ must exist in $\mathcal{U}$ and

$$
\frac{\dot{R}}{\dot{u}} \leqslant 0 .
$$

- From 2. Conditions (55) imply that

$$
\frac{\dot{\bar{m}}}{\dot{\bar{u}}} \leqslant 0
$$

in $\overline{\mathcal{U}}$. Therefore, under these circumstances, WEC's hold in $\overline{\mathcal{U}}$.

Taking into account (46) or (50), we obtain

$$
m(u<0, R>0) \stackrel{\Sigma_{\mathrm{SF}}}{>} 0 \quad \text { and } \quad m(u=0, R=0)=0 .
$$

In interiors in which $m(u, R=0)=0$ condition (60) implies $m,{ }_{R}(u, R)>0$ in $\mathcal{U} .^{5}$

${ }^{5}$ Note that the first two items help to delimit $\mathcal{U}$ and $\overline{\mathcal{U}}$. 
- From 3. If we define

$$
\eta \equiv \lim _{\lambda \rightarrow 0^{-}} \frac{\dot{R}}{\dot{u}}
$$

and

$$
\chi_{0 \eta}=\lim _{\lambda \rightarrow 0^{-}}\left(1-\frac{2 m}{R}\right),
$$

then the timelike condition in the interior leads us to

$$
\lim _{\lambda \rightarrow 0^{-}}\left(-\varepsilon \frac{\dot{R}}{\dot{u}}+\frac{\chi \mathrm{e}^{2 \beta}}{2}\right)=-\varepsilon \eta+\frac{\chi_{0 \eta}}{2} \geqslant 0 .
$$

By the way, note that the matching conditions imply that $\chi_{0 \eta}=\chi_{0 \zeta}$. So that, from (19), we will have $0<\chi_{0 \eta} \leqslant 1$ if the matching hypersurface is of type $\Sigma_{\mathrm{SF}}$.

\section{Constructing specific evaporating models with $\Sigma$ of type $\Sigma_{\mathrm{SF}}$}

In this section, first we will deal with some general considerations to see how the collapsing conditions together with the matching conditions restrict the different possible interiors. Then, we will analyse the consequences for every different type of interior.

In what follows it will be useful to define

$$
K_{0}^{ \pm} \equiv \lim _{\lambda \rightarrow 0^{-}} \frac{\dot{u}}{\dot{\bar{u}}}
$$

where \pm refers to the two possibilities $\varepsilon= \pm 1$.

We can write $\eta$ and $K_{0}^{ \pm}$as functions of the exterior quantities $\zeta$ and $\Upsilon$ if we use (47) or (51) (when $\epsilon=-1$ or +1 , respectively) and (13) as follows.

- If $\epsilon=-1$ then

$$
\eta=\zeta^{-1}, \quad K_{0}^{-}=1
$$

- If $\epsilon=+1$ then

$$
\eta=\frac{1-2 \Upsilon \zeta}{2+\zeta-2 \Upsilon \zeta^{2}}, \quad K_{0}^{+}=\frac{2+\zeta-2 \Upsilon \zeta^{2}}{\zeta-2 \Upsilon \zeta^{2}}
$$

It will be useful to note that $K_{0}^{ \pm}$are finite. This is obvious for $K_{0}^{-}$and can be shown for $K_{0}^{+}$using (5) and (18) since one gets $0 \leqslant K_{0}^{+} \leqslant 1$.

In order to compute $\Upsilon$ partially in terms of interior magnitudes we have

$$
\begin{aligned}
\Upsilon & \equiv \lim _{\bar{u} \rightarrow 0^{-}} \frac{\mathrm{d} \bar{m}}{\mathrm{~d} \bar{u}}=\lim _{\lambda \rightarrow 0^{-}} \frac{\dot{\bar{m}}}{\dot{\bar{u}}}=\lim _{\lambda \rightarrow 0^{-}} \frac{\dot{m}}{\dot{u}} \frac{\dot{u}}{\dot{\bar{u}}} \\
& =K_{0}^{ \pm}\left(m,{ }_{u}(0,0)+m,,_{R}(0,0) \eta\right) .
\end{aligned}
$$

\subsection{General considerations}

Let us now explore the consequences on the interior of including the evolution equations (48) or (52).

In what follows it will be useful to define

$$
A_{0} \equiv \lim _{\lambda \rightarrow 0^{-}} \frac{m,_{R}}{R \beta,,_{R}}
$$

Note that, in general, $A_{0}$ will depend on the particular matching hypersurface $\Sigma_{\mathrm{SF}}$. 
7.1.1. Case $\varepsilon=-1$. In this case $\chi_{0 \eta}$ and $\eta$ are finite, according to (18), (19) and (65). Then $A_{0}$ is also finite since from (48) we have

$$
\eta \equiv \lim _{\lambda \rightarrow 0^{-}} \frac{\dot{R}}{\dot{u}}=\lim _{\lambda \rightarrow 0^{-}}\left(\frac{m, R}{2 R \beta, R}-\chi\right)=\frac{1}{2} A_{0}-\chi_{0 \eta} .
$$

It is important to remark here that equation (69) can be in fact a nonlinear algebraic equation for the variable $\eta$.

Provided that $\eta<0$, equation (69) can be integrated in $\mathcal{U}$ in order to obtain

$$
R_{\mathrm{SF}} \sim \eta u \text {. }
$$

The timelike character of $\Sigma_{\mathrm{SF}}(63)$ together with (69) implies

$$
A_{0}-\chi_{0 \eta} \geqslant 0 \text {. }
$$

If (71) holds then $\Sigma_{\mathrm{SF}}$ will be timelike in $\mathcal{U}-\{u=R=0\}$.

Finally, conditions (71) and $\eta<0$ (from (18) and (65)) lead to

$$
\chi_{0 \eta} \leqslant A_{0}<2 \chi_{0 \eta} \text {. }
$$

7.1.2. Case $\varepsilon=+1$. In this case, from (52),

$$
\eta \equiv \lim _{\lambda \rightarrow 0^{-}} \frac{\dot{R}}{\dot{u}}=\lim _{\lambda \rightarrow 0^{-}} \frac{\chi\left(2 R \chi \beta,,_{R}-m,,_{R}\right)+2 m,_{u}}{2\left(R \chi \beta,,_{R}-m,,_{R}\right)} .
$$

The timelike character of the matching hypersurface is trivially satisfied if $\eta<0$ and $\chi_{0 \eta}>0$ in $\mathcal{U}$ since they imply $\lim _{\lambda \rightarrow 0^{-}}\left(-\dot{R} / \dot{u}+\chi \mathrm{e}^{2 \beta} / 2\right)>0$.

The condition $\eta<0$ is equivalent to

$$
\lim _{\lambda \rightarrow 0^{-}} \frac{\chi\left(2 R \chi \beta,,_{R}-m,,_{R}\right)+2 m, u}{2\left(R \chi \beta,,_{R}-m,,_{R}\right)}<0 .
$$

If $\eta$ is finite and negative then the integration of (73) in $\mathcal{U}$ provides

$$
R_{\mathrm{SF}} \sim \eta u \text {. }
$$

\subsection{Models with a regular interior}

In this subsection we would like to study the local behaviour of models with a regular interior that evaporate without creating an exterior singularity. A typical Penrose's diagram corresponding to a completely evaporating star of this type is illustrated in figure 6.

In this case, if we use (37) and (38) and the finiteness of $K_{0}^{ \pm}$on (67), then we get

$$
\Upsilon=0 \text {. }
$$

Since proposition 3.2 applies to a regular interior (38)

$$
\chi_{0 \eta}=1 \text {. }
$$

\subsubsection{Case $\varepsilon=-1$.}

Proposition 7.1. All conditions for an evaporating collapsing model with a regular interior will be fulfilled if, and only if, the interior satisfies $m(u<0, R) \stackrel{\Sigma_{\mathrm{SF}}}{>} 0$ in $\mathcal{U}-\{u=0, R=0\}$ and

$$
1 \leqslant \lim _{\lambda \rightarrow 0^{-}} \frac{m, R}{R \beta, R}<2
$$




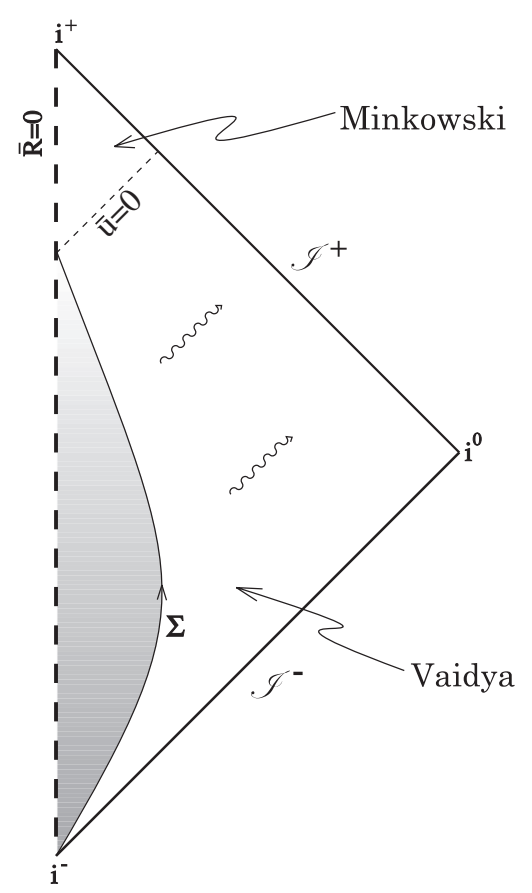

Figure 6. Penrose's diagram corresponding to a completely evaporating star. In this case the star's surface reaches $R=0$ and, simultaneously, the total mass vanishes completely.

This is easy to show by mere inspection of the conditions and taking into account that the last inequality comes from (72) for the particular case of a regular interior.

An example involving the lowest order derivatives of $m$ and $\beta$ that can be non-null at $u=R=0$ in the case of a regular interior (that is, we assume $m,{ }_{R R R}(0,0) \neq 0$ and $\beta, R R(0,0) \neq 0)$ is

$$
\eta \equiv \lim _{\lambda \rightarrow 0^{-}} \frac{\mathrm{d} R}{\mathrm{~d} u}=\frac{m, R R R(0,0)}{4 \beta, R R(0,0)}-1 .
$$

In $\mathcal{U}$ we can choose the integration constant in order to satisfy (53) and (54) so that the evolution equation can be written as

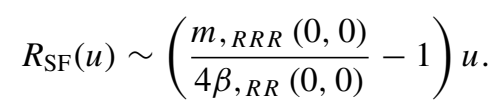

We have in this case

$$
\begin{aligned}
& m \stackrel{\Sigma}{\sim} \frac{1}{6} \dot{u}_{0}^{3} m, R R R(0,0) \eta^{3} \lambda^{3}>0, \\
& \beta \stackrel{\Sigma}{\sim} \frac{1}{2} \dot{u}_{0}^{2} \beta, R R(0,0) \eta^{2} \lambda^{2},
\end{aligned}
$$

in $\mathcal{U}$, where $\dot{u}_{0} \equiv \dot{u}(\lambda=0)$ is an arbitrary positive constant. Positivity of the mass function on $\Sigma_{\mathrm{SF}}$ implies $m_{R R R}(0,0)>0$ and therefore, condition (78) becomes

$$
0<2 \beta,_{R R}(0,0) \leqslant m,_{R R R}(0,0)<4 \beta,_{R R}(0,0) .
$$




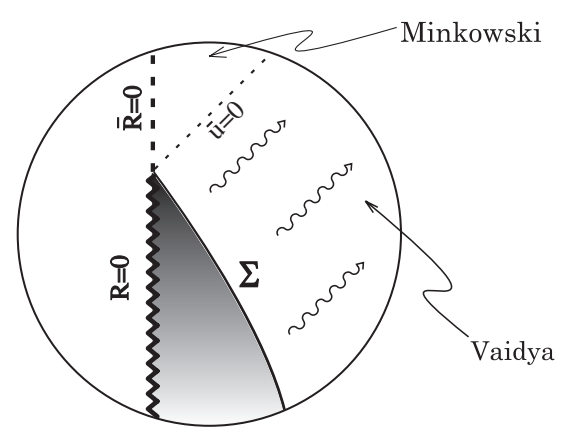

Figure 7. A stellar model with a pre-existing timelike curvature singularity completely evaporates. Therefore, a locally naked singularity is already present before the evaporation event. The figure only shows the behaviour around the total evaporation.

\subsubsection{Case $\varepsilon=+1$.}

Proposition 7.2. All conditions for an evaporating collapsing model with a regular interior will be fulfilled if, and only if, the interior satisfies $m(u<0, R) \stackrel{\Sigma_{\mathrm{SF}}}{>} 0$ in $\mathcal{U}-\{u=0, R=0\}$ and

$$
\lim _{\lambda \rightarrow 0^{-}} \frac{\left(2 R \beta,_{R}-m,_{R}\right)+2 m,_{u}}{2\left(R \beta,_{R}-m,_{R}\right)}<0 .
$$

The last inequality comes from (74) applied to the regular interior case.

The first example involving the lowest non-null derivatives of $m$ and $\beta$ is

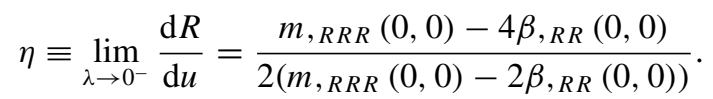

The functions $m(\lambda)$ and $\beta(\lambda)$ are expressed in $\mathcal{U}$ as in (81) and (82), respectively. Positivity of the mass function on $\Sigma_{\mathrm{SF}}$ implies $m, R R R(0,0)>0$ and therefore, condition (84) becomes

$$
0<2 \beta,_{R R}(0,0) \leqslant m,_{R R R}(0,0)<4 \beta,_{R R}(0,0) .
$$

\subsection{Models with a non-spacelike interior singularity}

We will now consider interiors with $m(u, 0)=0$ and $m,_{R}(u, 0) \leqslant 1 / 2$, but not satisfying all the conditions for a regular interior (38), what implies the existence of a non-spacelike singularity at $u=R=0$. The interior of these models must satisfy

$$
m,_{R}(0,0)=0 .
$$

To show this in the case $\varepsilon=-1$ it is enough to consider the finiteness of $A_{0}$ and its definition (68). In the case $\varepsilon=+1$ we must take into account that these models have $m,{ }_{u}(0,0)=0$ and the negativeness of $\eta$ in (73) to show it.

On the other hand, (87) implies that $\Upsilon=0$ and $\chi_{0 \eta}=1$ (see the previous subsection). Since the existence of a null singularity at $R=0$ requires $m,_{R}(u, 0)=1 / 2$, it is clear that an interior spacetime with a null singularity at $R=0$ cannot be matched with a Vaidya metric across a timelike hypersurface.

The Penrose diagram around the evaporation event for this case is illustrated in figure 7. 
7.3.1. Case $\varepsilon=-1$. Similarly to the regular interior case we have the following.

Proposition 7.3. All conditions for an evaporating collapsing model with a non-spacelike interior singularity will be fulfilled if, and only if, the interior satisfies $m(u<0, R) \stackrel{\Sigma_{\mathrm{SF}}}{>} 0$ in $\mathcal{U}-\{u=0, R=0\}$ and

$$
1 \leqslant \lim _{\lambda \rightarrow 0^{-}} \frac{m, R}{R \beta,,_{R}}<2
$$

An example with the possible lowest order derivatives that could be non-null at $u=R=0$ would be

$$
\eta \equiv \lim _{\lambda \rightarrow 0^{-}} \frac{\mathrm{d} R}{\mathrm{~d} u}=\frac{m,_{u R}(0,0)+m,_{R R}(0,0) \eta}{2 \beta,_{R}(0,0) \eta}-1
$$

Now, $\eta$ is a negative solution of the previous quadratic algebraic equation.

We have in this case

$$
\begin{aligned}
& m \stackrel{\Sigma}{\sim} \frac{1}{2} \dot{u}_{0}^{2}\left(2 m,_{u R}(0,0)+m,_{R R}(0,0) \eta\right) \eta \lambda^{2}, \\
& \beta \stackrel{\Sigma}{\sim} \dot{u}_{0} \beta,_{R}(0,0) \eta \lambda,
\end{aligned}
$$

in $\mathcal{U}$. Positivity of mass function on $\Sigma_{\mathrm{SF}}$ implies $2 m,{ }_{u R}(0,0)+m, R R(0,0) \eta<0$.

Finally, condition (88) becomes

$$
1 \leqslant \frac{m,_{u R}(0,0)+m,_{R R}(0,0) \eta}{\beta,_{R}(0,0) \eta}<2 .
$$

\subsubsection{Case $\varepsilon=+1$.}

Proposition 7.4. All conditions for an evaporating collapsing model with a non-spacelike interior singularity will be fulfilled if, and only if, the interior satisfies $m(u<0, R) \stackrel{\Sigma_{\mathrm{SF}}}{>} 0$ in $\mathcal{U}-\{u=0, R=0\}$ and

$$
\lim _{\lambda \rightarrow 0^{-}} \frac{\left(2 R \beta,_{R}-m,_{R}\right)+2 m,_{u}}{2\left(R \beta,_{R}-m,_{R}\right)}<0 .
$$

The last inequality comes from (74) applied to the case with a non-spacelike interior singularity.

The corresponding example involving the lowest order derivatives that could be non-null in this case implies

$\eta \equiv \lim _{\lambda \rightarrow 0^{-}} \frac{\mathrm{d} R}{\mathrm{~d} u}=\frac{\left(m,{ }_{R R}(0,0)-2 m,_{u R}(0,0)-2 \beta,,_{R}(0,0)\right) \eta+m,_{u R}(0,0)}{2[(m, R R}$.

Therefore, $\eta$ is a negative solution of this quadratic algebraic equation.

The functions $m(\lambda)$ and $\beta(\lambda)$ are expressed in $\mathcal{U}$ as in (90) and (91), respectively. Positivity of mass function on $\Sigma_{\mathrm{SF}}$ implies $2 m_{u R}(0,0)+m_{R R}(0,0) \eta<0$.

Finally, condition (93) is directly

$$
\frac{\left(m,,_{R R}(0,0)-2 m,_{u R}(0,0)-2 \beta,_{R}(0,0)\right) \eta+m,_{u R}(0,0)}{2[(m, R R}<0 .
$$




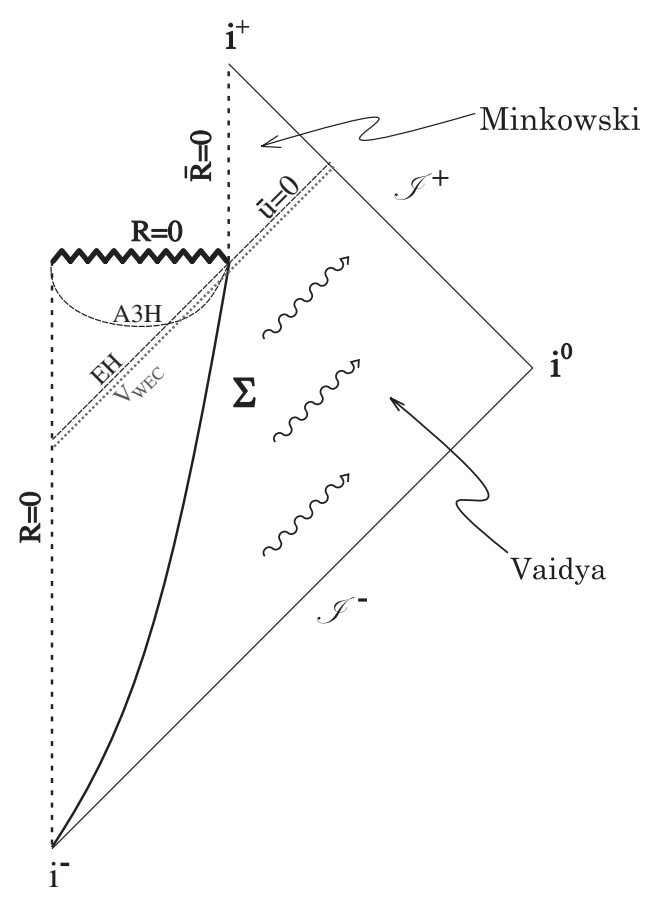

Figure 8. Typical Penrose's diagram for an evaporating star with an inner black hole region. Note that the outgoing null radial geodesic labelled $\bar{u}=0$ emerges from the singularity and reaches the future null infinity. Therefore, there is an instantaneous globally naked singularity in this situation. The radial outgoing null geodesic outside the $\mathrm{BH}$ labelled as $V_{\mathrm{WEC}}$ has a positive expansion from $R=0$ until reaching the $\mathrm{A} 3 \mathrm{H}$. After crossing it, the expansion becomes negative and the geodesic continues its travel to the future null infinity crossing again the $\mathrm{A} 3 \mathrm{H}$ and turning its expansion to positive again. Therefore [19], it must traverse regions where the weak energy conditions are violated.

\subsection{Interiors with spacelike singularities}

We would like to analyse those models describing the evaporation of a star in which a spacelike singularity $\left(m(u<0, R=0)>0\right.$ or $m(u<0, R=0)=0$ but $\left.m,{ }_{R}(u<0, R=0)>1 / 2\right)$ and a black hole interior region are already present.

These models must obey the minimum set of conditions (subsection 6.1). Furthermore, as we discussed then, around the spacelike singularity $\chi<0$ and, thus, the existence of a black hole region demands $\varepsilon=+1$. Another less usable condition is that in order to have a total evaporation $(m(u=0, R=0) \stackrel{\Sigma}{=} \bar{m}(\bar{u}=0)=0)$ the star's surface cannot cross the event horizon. A typical Penrose's diagram of the complete matched spacetime for this situation is shown in figure 8.

Note that there are radial outgoing null geodesics ending at the $R=0$ singularity so that their expansion is initially positive and becomes negative once they cross the $\mathrm{A} 3 \mathrm{H}$ (section 3 ). The EH is one of these null geodesics. Likewise, the degree of differentiability of the spacetime guarantees that there are radial outgoing null geodesics close enough to the EH (and to its right-see figure 8) with an initial positive expansion that becomes later negative. These null geodesics do not end at the singularity, but continue their travel to the future null infinity and must, therefore, turn their expansion to positive again, after crossing the A3H somewhere in 
the stellar interior. This, according to [19] (proposition 9.2.8), can only happen if they traverse regions where the weak energy conditions are violated. Let us remark that despite figure 8 illustrates a particular case of evaporating black hole, the behaviour of the $\mathrm{EH}$ and the $\mathrm{A} 3 \mathrm{H}$ around the total evaporation event is totally generic. Thus, all these evaporating black holes must violate the WECs.

In this case, the conditions explained in subsection 6.1 for $\varepsilon=+1$ lead us to a rather complex set of inequalities that must be fulfilled. Therefore, in order to show the existence of solutions modelling evaporating black holes, we will just work out a particular example. Let us consider $m(u, R) \simeq m_{40} u^{4}+m_{03} R^{3}$ and $\beta(u, R) \simeq \beta_{02} R^{2}$ in $\mathcal{U}$, where $m_{40}, m_{03}$ and $\beta_{02}$ are constants. Using the procedure described above taking into account that $\varepsilon=+1$ we find

$$
\eta^{-1}=\frac{2\left(3 m_{03}-2 \beta_{02}\right)}{3 m_{03}-4 \beta_{02}}
$$

If we now take into account the non-negativity of the exterior mass function and the negativity of $\eta$ (what also guarantees a timelike $\Sigma$ ) we get the following set of conditions for the correct behaviour of $\Sigma$ :

$$
0<2 \beta_{02} \leqslant 3 m_{03}<4 \beta_{02}
$$

On the other hand,

$$
\frac{\mathrm{d} \bar{m}(\bar{u})}{\mathrm{d} \bar{u}} \simeq-3 m_{03}\left(1-\frac{3 m_{03}}{4 \beta_{02}}\right) R^{2} .
$$

In this way, $\Upsilon=0$.

\section{Generation of exterior light-like singularities}

If a star collapses and an exterior light-like singularity at $\bar{R}=\bar{u}=0$ appears then the timelike matching hypersurface must be of type $\sigma_{\mathrm{NS}}$ and, thus, (16) and (17) must be fulfilled. From (16) we know that for this case $\zeta=\xi_{0+}$, so we can easily find $\zeta$ using (7). We have to consider two different cases according to the two different values for $\epsilon$.

- Case $\epsilon=-1$. The value of $\eta$ can be obtained from (65):

$$
\eta=(\sqrt{1+16 \Upsilon}-1) / 4=-\frac{\chi_{0 \eta}}{2} .
$$

Equation (69) together with (99) implies $A_{0}=\chi_{0 \eta}$.

- Case $\epsilon=+1$. The value of $\eta$ can be obtained from (66):

$$
\eta=-\infty \text {. }
$$

This, together with the differentiability of the metric, the limits on $\chi_{0 \eta}$ (17) and (73), implies $A_{0}=\chi_{0 \eta}$.

Therefore, in both cases, $A_{0}$ must be a finite quantity satisfying $0 \leqslant A_{0} \leqslant 1 / 2$ according to (17). Since $\lim _{\lambda \rightarrow 0^{-}} R=0$, the definition of $A_{0}$ (68) implies that

$$
m,_{R}(0,0)=0 .
$$

We now consider the possibility that $m(u, R=0)=0$ on a neighbourhood of $u=R=0$, then (101) would imply $\chi_{0 \eta}=\lim _{\lambda \rightarrow 0^{-}} \chi=1$ (according to proposition 3.2 ), what contradicts the necessary requirement (17) for the matching with a $\Sigma_{\mathrm{NS}}$ hypersurface. The only possibility left for a total evaporation is that near the evaporation event $m(u<0, R=0)>0$ and $m(u=0, R=0)=0$. Let us consider the two possibilities for $\epsilon$. 
- If $\epsilon=-1$ and $m(u<0, R=0)>0$, then there is an inner spacelike singularity and the 2-spheres near to it will be closed surfaces trapped to their past (i.e. there is a white-hole-like region), what we think can be discarded for a stellar interior. However this possibility has been already treated for the particular (non-stellar) case in which the interior is Vaidya's spacetime, as can be seen in figure 3, if we take for the matching hypersurface $\sigma_{\mathrm{NS}}$.

- If $\epsilon=+1$ and $m(u<0, R=0)>0$ the model must have a previous inner spacelike singularity, but now the 2-spheres near to it will be closed surfaces trapped to their future (i.e. there would be a black-hole-like region). On the other hand, $\chi_{0 \eta}$ can be easily written as

$$
\chi_{0 \eta}=1-2 \lim _{\lambda \rightarrow 0^{-}}\left(m,,_{R}+m, \frac{\dot{u}}{\dot{R}}\right) .
$$

Taking into account the differentiability of the mass function, (100) and (101), it is clear that $\chi_{0 \eta}=1$, which contradicts (17).

The considerations above can be summarized in the following.

Proposition 8.1. A collapsing star endowed with an interior $C^{2}$ mass function cannot develop an exterior light-like singularity at the total evaporation event.

\section{Discussion and conclusions}

Due to the great number of situations in which the evaporating models have been used in the current literature, our aim in this paper was to study the evaporating models as a whole and to analyse their common properties, behaviours and possibilities. Our work was centred on the evaporating event itself. However, we have to remark that this semiclassical study is not completely general. This is due to the fact of demanding an interior $C^{2}$ metric in $\mathcal{U}$. This, for example, prevents us to include in our study singular evaporating models in which the mass function is not even well defined at $u=R=0$ (see, for instance, [1]. ${ }^{6}$ ) Taking this into account, let us now list our main results.

First, we have treated stellar models that evaporate without reaching a complete collapse, i.e. models in which the star simply gets rid of all its mass in a more or less violent process. Proposition 5.1 tells us that whenever the dominant energy conditions are fulfilled and in the absence of closed trapped 2-spheres there must be an outgoing spherically symmetric light-like hypersurface crossing the matched spacetime such that the mass function is null on it and to its future.

As stated in the introduction the evaporating models have been often used in the current literature as a means of avoiding the generation of singularities during the collapse (from [7] to [12]), sometimes under the name of incipient BHs or non-singular evaporating BHs (from [13] to [18]). In this respect, we have shown (propositions 7.1 or 7.2) that a collapsing stellar model with a regular interior will evaporate if, and only if, the mass function on the matching hypersurface is non-negative and condition (78) (or 84) is satisfied for the $\varepsilon=-1$ (or the $\varepsilon=+1$, respectively) case. Furthermore, provided that the conditions are fulfilled, we have shown that the star cannot generate an exterior light-like singularity (proposition 8.1) such as the one illustrated in figure 9. This is remarkable because, even if such situation is not possible in non-radiative models (Schwarzschild's exterior), it seemed feasible with an exterior radiating Vaidya's solution. In this way, the complete matched model must be singularity free. Figure 6 illustrates this situation.

6 Nevertheless, some of our results apply. For example, in the aforementioned model $\chi_{0 \eta}=1$ and, therefore, according to (17) it cannot develop an exterior singularity. 


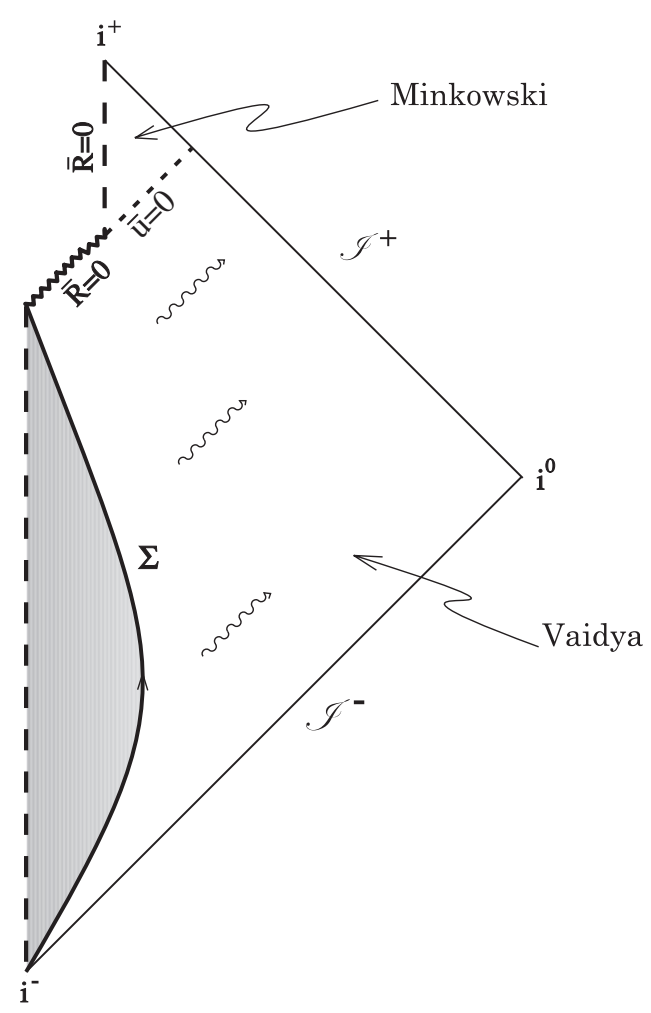

Figure 9. Hypothetical Penrose's diagram corresponding to a completely evaporating star with a regular interior. We have shown that this possibility is not feasible under our assumptions since an exterior light-like singularity cannot appear.

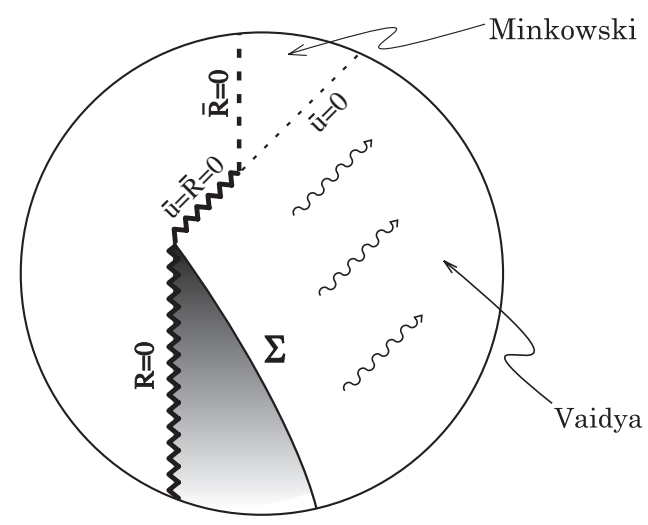

Figure 10. Hypothetical Penrose's diagram around the evaporation event corresponding to a completely evaporating star with a interior timelike singularity. We have shown that this possibility is not feasible under our assumptions since an exterior light-like singularity cannot appear.

We have extended our study to stellar models in which an inner non-spacelike singularity (and, therefore, naked) has already formed during the collapse. In this case, we have shown 


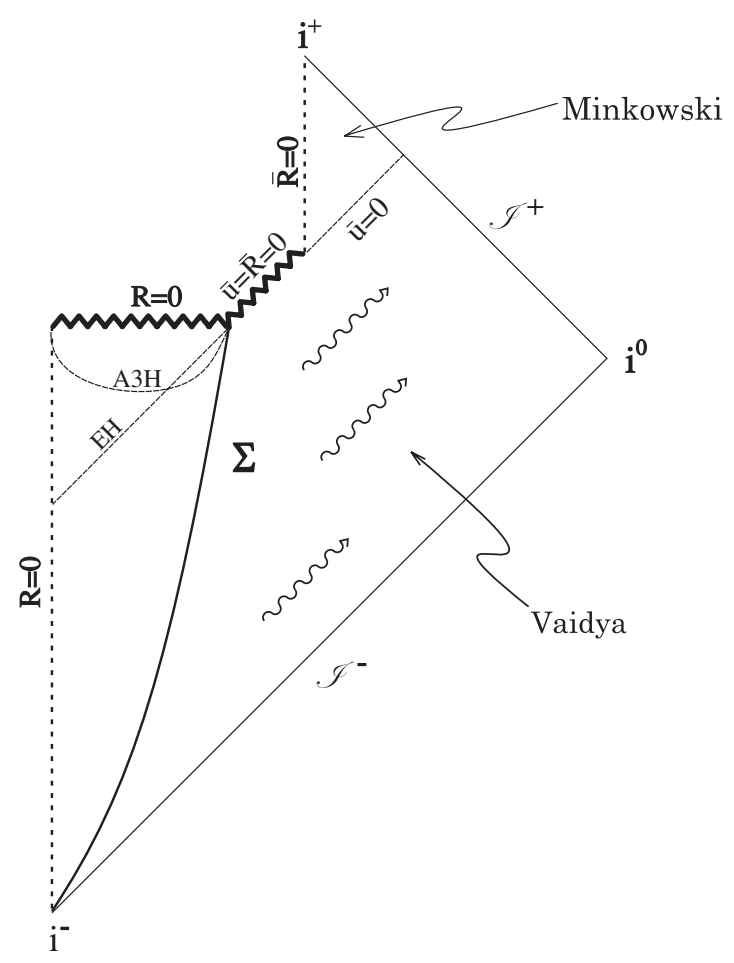

Figure 11. Hypothetical Penrose's diagram for an evaporating star that collapses creating an inner black hole region and an exterior light-like singularity. We have shown that this possibility is not feasible under our assumptions since an exterior light-like singularity cannot appear.

(propositions 7.3 or 7.4) that a collapsing stellar model with an inner non-spacelike singularity will evaporate if, and only if, the mass function on the matching hypersurface is non-negative and condition (88) (or (93)) is satisfied for the $\varepsilon=-1$ (or the $\varepsilon=+1$, respectively) case. Furthermore, provided that the conditions are fulfilled, we have shown that the star cannot generate an exterior light-like singularity (proposition 8.1) such as the one illustrated in figure 10. In this way, the evaporation process seems to evaporate the inner singularity in the case of not being spacelike. In other words, the evaporation process eliminates any pre-existing naked singularity. Figure 7 illustrates this situation around the evaporation event.

Finally, we have treated the case in which an inner spacelike singularity pre-exists. The models presented here can be considered as a special type of evaporating black hole since the object achieves the total collapse with null total mass. These models violate the weak energy conditions and they all posses an instantaneous naked singularity that does not extend to the exterior of the collapsing object (figure 11). We have illustrated this type of evaporating black holes in figure 8 .

\section{Acknowledgments}

The authors would like to acknowledge the Ministerio de Educación y Ciencia (FIS200763034) and the Generalitat de Catalunya (grant 2005SGR 00515) for financial support. 


\section{References}

[1] Demianski M and Lasota J P 1968 Astrophys. Lett. 1205

[2] Steinmüller B, King A R and Lasota J P 1975 Phys. Lett. A 51191

[3] Penrose R 1969 Riv. Nuovo Cimento 1252

Joshi P S 2002 Mod. Phys. Lett. A 17 15-7 1067 (for a recent review)

[4] Lake K and Hellaby C 1981 Phys. Rev. D 243019

[5] Unruh W G 1985 Phys. Rev. D 312693

Lake K and Hellaby C 1985 Phys. Rev. D 312695

[6] Banerjee A, Chatterjee S and Dadhich N 2002 Mod. Phys. Lett. A 172335

Banerjee A and Chatterjee S 2005 Astrophys. Space Sci. 299219

[7] Fayos F, Senovilla J M M and Torres R 1996 Phys. Rev. D 544862

[8] Fayos F and Torres R 2008 Class. Quantum Grav. 25175009

[9] Hirschmann E W and Eardley D M 1995 Phys. Rev. D 514198

[10] Schäfer D and Goenner H F 2000 Gen. Rel. Grav. 322119

[11] Chang Z, Guan C-B, Huang C-G and Li X 2008 Int. J. Theor. Phys. 472479

Chang Z, Guan C-B, Huang C-G and Li X 2008 Commun. Theor. Phys. 50271

[12] Joshi P S and Goswami R 2007 Class. Quantum Grav. 242917

[13] Gerlach U H 1976 Phys. Rev. D 141479

[14] Hayward S A 2006 Phys. Rev. Lett. 96031103

[15] Vachaspati T and Stojkovic D 2007 Phys. Rev. D 76024005

[16] Barceló C, Liberati S, Sonego S and Visser M 2008 Phys. Rev. D 77044032

[17] Ashtekar A and Bojowald M 2005 Class. Quantum Grav. 223349

[18] Bonanno A 2006 Phys. Rev. D 73083005

[19] Hawking S W and Ellis G F R 1973 The Large Scale Structure of Space-Time (Cambridge: Cambridge University Press)

[20] Vaidya P C 1951 Proc. Indian Acad. Sci. A 33264

[21] Bondi H, van der Burg M G J and Metzner A W K 1962 Proc. R. Soc. A 26921

[22] Hernández W C and Misner C W 1966 Astrophys. J. 143452

[23] Cahill M E and McVittie G C 1970 J. Math. Phys. 111382

[24] Zannias T 1990 Phys. Rev. D 413252

[25] Perko L 2001 Differential Equations and Dynamical Systems (New York: Springer)

[26] Tricomi F G 1961 Differential Equations (London: Blackie and Sons)

[27] Volovich I V, Zagrebnov V A and Frolov V P 1976 Teor. Mat. Fiz. 29191

[28] Papapetrou A 1985 A Random Walk in General Relativity (New Delhi: Wiley Eastern)

[29] Dwivedi I H and Joshi P S 1989 Class. Quantum Grav. 61599

[30] Carminati J and McLenaghan R G 1991 J. Math. Phys. 323135

[31] Torres R 2001 Construcción de modelos de estrellas y burbujas de vacío o radiativas, en el marco de la Relatividad General: Un planteamiento global. PhD Thesis Universitat Politècnica de Catalunya, Barcelona

[32] Fayos F, Senovilla J M M and Torres R 2003 Class. Quantum Grav. 202579

[33] Mars M and Senovilla J M M 1993 Class. Quantum Grav. 101865 